\title{
Modeling the Multi-channel Section in the Supply Chain System using the Multiserver Queue Analogy
}

\author{
Petrus Setya Murdapa ${ }^{1^{*}}$
}

\begin{abstract}
The industry generally consists of a supply chain system. The main constituents of any supply chain system are suppliers, manufacturers, distribution centers, and retailers. The system configuration can be straight chain, branched, cyclic, or a combination of all. An analytical model is needed to study system behavior as a result of the dynamics of its constituents. Modeling a multi-channel section becomes quite a challenging job in this regard. A method of modeling the multi-channel section will be discussed in this paper by adopting multiserver queues. As is well known, in a multi-server queue, there is a branching point at which the flow of entities begins to spread across several parallel servers. In the modeling perspective of this paper, the branching point is in the buffer (finished good warehouse in the factory, i.e., the focal echelon). That is the end of the waiting line from which the entity specifically moves to one of the servers, or in this context; it is called a channel. In this paper, the number of channels can be any, generalizable, can be more than two. Hence, the subsystem studied includes a factory, finished product warehouse, and several distribution centers. The factory produces by the mechanism of, where and $\mathrm{r}$ are stopping point and production restarting point, respectively. Production stops when the quantity of finished product in the warehouse reaches units and will restart the production when the quantity drops to the same or lower than units. The model is developed under Markovian assumptions by considering the quantities of production rates, the number of distribution centers (channels), travel time from factories to each distribution center, delivery lot size, and the time between the arrival of orders from distribution centers. The system under study is seen as a case of two echelons, namely factories and distribution channels. The numerical model obtained is applied to one case example with certain conditions. Comparisons with discrete simulation results give relatively small and acceptable differences. So, in the future, this model can complement the overall modeling of the supply chain system, a multi-echelon system with multi-channel distribution.
\end{abstract}

Keywords: Supply chain, multi channel distribution, production restarting point, production stopping time, markovian model.

\section{Introduction}

A supply chain system is composed of a series of supplier-factory-distributor-retailer. Each of those subjects in the supply chain is generally a company with management independent from one another. However, they will collaborate to obtain a smooth process for supplying products to end consumers. The number of suppliers on the upstream side of the factory and the number of distribution channels on the downstream side is generally more than one (branching supply chain).

Efficiency is one of the crucial determinants of industrial sustainability. It is necessary to have good cooperation between subjects in the supply chain system. Such cooperation must be based on high integrity, in the spirit that the supply process must create

${ }^{1}$ Faculty of Engineering, Department of Industrial Engineering, Univesitas Katolik Widya Mandala Surabaya, Jl. Manggis 15-17 Madiun, Indonesia 60238. Email: petrus.setya@ukwms.ac.id

* Corresponding author the competitiveness of the products distributed. Various studies have shown that a holistic review of the stock items in the system will minimize the total cost of inventory rather than a partial review of the respective subjects. This study shows the importance of supply chain management and its intended integrity.

Various models of the supply chain have been described in the literature, either in optimization models or performance models. Optimization models are more often expressed in linear mathematical programming, while performance models are in simulation models or queuing network models. The performance model for the supply chain system is quite similar to the performance model for the production line system. There are two types of production line systems, namely, flow lines and transfer lines. The supply chain system can be analogized to a flow line system [1]. Here, processing at each workstation is assumed to be exponentially distributed. The model was built using decomposition techniques. Several stations in the system are aggregated to form several 

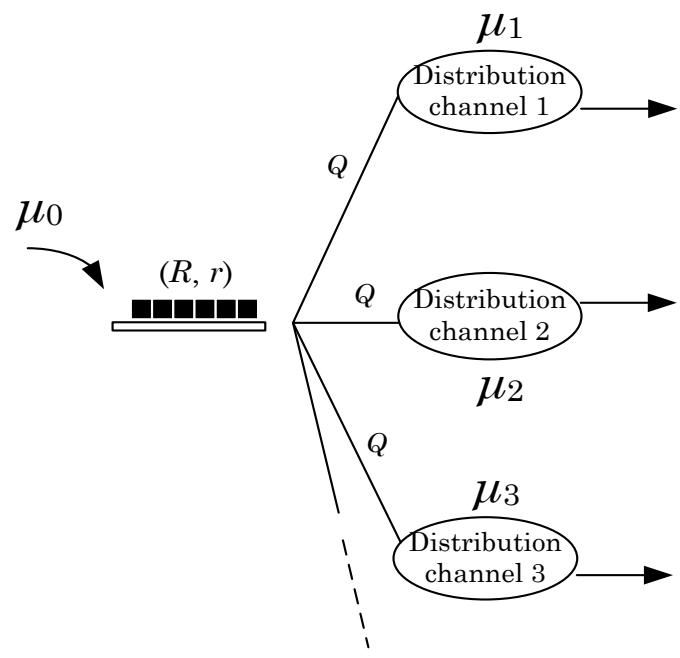

Q

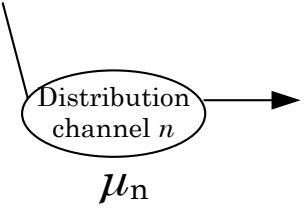

$\mu_{0}:$ arrival rate (production rate)

$\mu_{\mathrm{i}}:$ service rate of distribution channel- $i$ where $i=2,3, \ldots, n$

$Q$ : distribution lotsize (the same for all channel)

Figure 1. A single-stage queuing system that is used as a model for a multi-channel distribution system (An extension of [1])

alternatives of a two-stage subsystem. Each stage is an aggregate stage, each representing the upstream and downstream sides. Overall, the original system looks as if it was decomposed into several two-stage subsystems.

Like flowlines, supply chain systems can also be viewed as queuing network systems. The only differrence is the distance between the stages. In a production line system, the distance between stages (i.e., workstations) from one to the next is very close. Whereas in the supply chain system, the distance between the stages (namely echelons) is very far (up to tens to hundreds of $\mathrm{km}$ ). The supply chain system is a series of sequential echelons that work together to fulfill a product to the end consumer. It can be identified as a flow line system if the echelons are seen as workstations. This is seen in Karaman and Altiok [2], Saetta et al. [3], and Murdapa et al [4].

Assuming that a supply chain is a flow line system, Murdapa et al [4] developed a supply chain system model with inventory control at each stage, as in [2]. Those studies give different subsystem structures when the decomposition analysis technique is applied. Meanwhile, Murdapa et al. [1] analogized a two-channel distribution with queues in front of two parallel servers.
A multiserver queuing system is still an interesting study. The phenomenon occurs in several fields with a variety of problems. Xiong and Altiok [5] studied multiserver queuing systems to model the maximum limit of waiting time available in an information processing system. Koroliuk et al. [6] and Pryshchepa et al. [7], for example, examine the problem of the multiserver queuing phenomenon in telecommunications issues where there is retrial arrival. Sultan et al. [8, 9] examined the problem of a multiserver system where arrivals occur in batches using the Montecarlo simulation method. Another form of multiserver system variation is revealed by Ke et al. [10] in a system where there are optional second type service facilities, and Goswami [11] discusses a system with arrival balking.

This paper adopts a simple multi-server queuing concept, where departures occur in batches to model distribution channeling. Products to be distributed are taken directly from the finished product warehouse at the factory. The factory itself monitors the availability of its product stock using the mechanism named as by Liberopoulos and Dallery [12]. The lotsizes of the distribution on each channel are assumed to be the same as each other, which is denoted as Figure 1. Hence, the proposed preliminary model in [1] was limited to two channels and has not been validated with simulation results. In this paper, the model is generalized to many channels and validated with simulation results.

In a real industry, the supply chain system will always have multiple distribution channels. A model for rapid analysis is needed to facilitate a broader discussion of the performance of a more general supply chain system. However, most models were formulated in the form of linear programming. The linear models ignore the dynamics of the states of the system. These states in the system will significantly affect system performance. A performance model based on how the system behaves by accommodating the dynamics of the existing states in the system will help simulate decision scenarios related to the system that is closer to the real system conditions. For this reason, it is essential to construct a model that focuses on the multi-channel section of the supply chain system in the type of performance model. In the future, the model will complement other sections' models, forming a complete overall model as the primary objective.

This paper generalizes the two-channel case of Murdapa et al. [1] to accommodate any number of channels. Products that are stored in FG Stock will be sent to more than two distribution channels. The duration of the existing processes is assumed to be exponentially distributed, the same as in [1]. The 


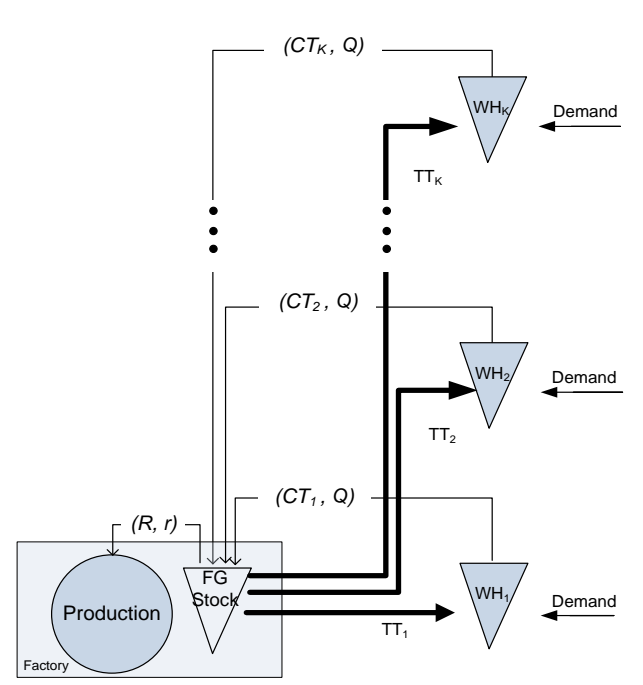

Figure 2. The system studied consists of the following echelons: factory $(R, r)$ and multi distribution channel

duration between two consecutive events is often modeled by a geometric, exponential, or other families of Markovian distribution [2,3,13,14,15]. The multi-channel distribution model will be very useful as a basis that will complement the supply chain system modeling on several distribution channels. For that purpose, a decomposition method could be used to approximate some good solutions, as in $[16,17]$. The decomposition techniques also could be used to analyze more extensive systems, i.e., supply chain (see [2,3,4]). There, the models in this paper would contribute to this field in the future.

\section{Methods}

This paper is outlined as follows. First, an overview of the hypothetical system under study is presented. The behavior and assumptions regarding the system will be described. Second, the quantities which are known, and which are to be calculated are notated. Third, system modeling is carried out under the assumption that the system is already in a steady state. The conceptualization of state dynamics is expressed in a state transitions diagram, where the system states are defined first. Then, the state transitions are formulated in equations. Next, a numerical calculation algorithm is compiled and written into computer programming so that experimentation can be carried out quickly. Finally, an evaluation and discussion are conducted regarding the numerical model and its calculation results.

\section{Description of the scope of the system under study}

The system discussed in this paper will be a part of a generic supply chain system. It will be a single-stage that consists of two echelons, namely the factory and distribution channel, as shown in Figure 2. The
Table 1. Lisf of notation

\begin{tabular}{|c|c|c|}
\hline$r$ & : & Reproduction point \\
\hline$R$ & $\cdot$ & Production stopping point \\
\hline$Q$ & : & $\begin{array}{l}\text { Distribution lotsize (the same for all } \\
\text { warehouses) }\end{array}$ \\
\hline$\mu_{0}$ & $:$ & Average production rate \\
\hline$\mu_{i}$ & & Delivery rate to warehouse- $i$ or channel- $i$ \\
\hline$T T_{1}$ & $:$ & $\begin{array}{l}\text { The average transportation leadtime to the } \\
\text { warehouse- } 1\end{array}$ \\
\hline$\vdots$ & & \\
\hline$T T_{i}$ & $:$ & $\begin{array}{l}\text { The average transportation leadtime to the } \\
\text { warehouse- } n\end{array}$ \\
\hline $\begin{array}{l}C T_{1} \\
\vdots\end{array}$ & $:$ & Average inventory cycle time in warehouse- 1 \\
\hline$C T_{i}$ & : & Average inventory cycle time in warehouse- $i$ \\
\hline$K$ & $:$ & $\begin{array}{l}\text { Number of channels (or number of } \\
\text { warehouses) }\end{array}$ \\
\hline$m$ & $:$ & $\begin{array}{l}\text { Positive integer constant to define } \\
\text { nonproduction pathways }\end{array}$ \\
\hline$k$ & $:$ & $\begin{array}{l}\text { Positive integer constant to define negative } \\
\text { inventory depth }\end{array}$ \\
\hline
\end{tabular}

factory is assumed to produce according to the mechanism of production activities. Production activities will restart periodically when the inventory level in the finished product warehouse, FG Stock, is less or equal to $r$. Moreover, the activity stops when the level has reached $R$. Thus, the mechanism will repeat. Products are sent in $Q$ units to each distribution channel according to their demands. Hence, the product entities will flow downstream according to the pull mechanism. Karaesmen and Dallery [18] discussed the pull mechanism in a production line, while Karaman and Altiok [2], Saetta et al [3] for supply chain case. Table 1 shows notation list of the known quantities (or input quantities) related to the system under study.

\section{Modeling}

The warehouse in the channel- $i$ in Figure 2 is represented as $W H_{i}$. There will be $K$ channels. Shipments from the factory to channel- $i$ will take an average of $T T_{i}$ unit time. Channel- $i$ will place the orders to the factory on average every $C T_{i}$ unit of time. Shipment to the channel- $i$ will occur every $T T_{i}+C T_{i}$ so that the shipment rate on the distribution channel- $i, \mu_{i}$, can be expressed as:

$\mu_{i}=\frac{Q}{T T_{i}+C T_{i}}$

In Figure 2, the echelons representing the distribution channel are not yet represented. Figure 2 can be expressed in the form of a conceptual diagram in Figure 3, where all distribution channel echelons have been clearly added (DCs) to accommodate the existence of distribution echelons. The shipment rate to the distribution channel echelon- $i$ is the inverse of the time between shipments on that channel. This paper focuses on the multi-channel distribution section, shown by the box bounded by the dashed 


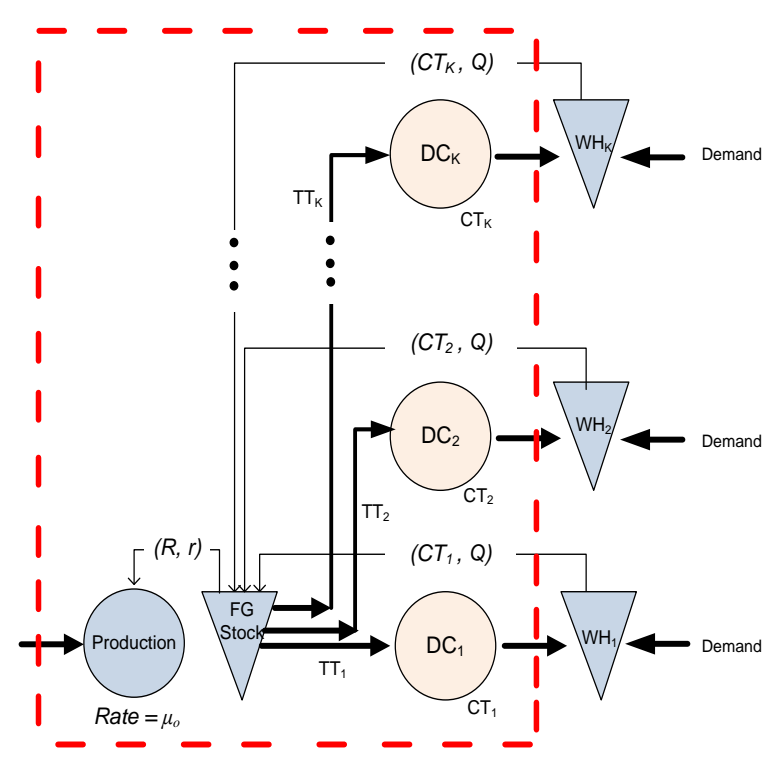

Figure 3. Conceptual diagram of the system under study (in a box with dashed-lines)

line. It is assumed simply that the shipment lot size to each distribution channel is the same, that is $Q$. The system is a single queue system with several parallel servers.

The system condition is described by the level of finished product inventory in FG Stock. State- $i$ where $i=0$ to $\infty$ (infinite). It means that the level of finished product inventory in FG Stock equal to $i$ units. With this definition, there will be many possible states. The maximum level in FG Stock is $R$. Backordering is assumed. Then the system condition can be negative, namely when the inventory level is below zero, which means that the factory has a backlog that must be met later. If the probability that the system is in state- $i$ is $P_{i}$, then a state transition diagram can be drawn up as in Figure 4. Because theoretically, the system can be in the state $i=-\infty$, so it is necessary to truncate the transition diagram only, not less than $i=R-k Q$ where $k$ is a large enough positive integer. In this case, the value of $k$ is determined at the beginning of the calculation. Ideally $k$ should be large enough, but not necessarily too large. Assigning $k$ that is too large will cause the computation time to be too long even though it does not affect the final value. An analytical solution would be too difficult to obtain. A numerical solution was used instead.

In the transition diagram, there are two transition paths, namely the production and the nonproduction path. On the production path, there will be a reduction in stock levels and also production activities. The nonproduction path occurs when the system reaches state $R$. At that state, production activity will stop, then the decrease in inventory level (state transitioning to the left) will follow the nonproduction path. The states that are in the nonproduction path are indicated by the addition of the notation $N$. For example, state $(N, R-Q)$ indicates a condition where there is a $R-Q$ units of product in FG Stock, and at that time there is no production activity. The state transition goes out of the nonproduction path when $R-m Q \leq r$ where $m$ is a constant positive integer. Murdapa et al [1, 4] proposed an approach to this case by omitting states which are incremented by the actual production lot that occurs one by one unit at a time according to the mechanism of $(R, r)$. Hence, here, state transitions jump in $Q$ units.

Under these simplification, if the input quantities, $r, R$ and $Q$ are given, then:

$r \geq R-m Q$ where $m$ is a positive integer

or the value of $m$ must be:

$m=\left\lceil\frac{R-r}{Q}\right\rceil$

where $\lceil x\rceil$ is the smallest integer that greater than $x$

A suitable transition diagram is shown in Figure 4, which is in principle the same as that depicted in Murdapa et al [1]. The difference is only in the number of channels represented by the left downward curved arrows, which in this study are more numerous. The state is divided into seven zones based on the transition pattern, namely zones I, II, IIIa, IIIb, IV, V, and VI. The zones represent the sectors on the state transition diagram, which are distinguished from one another by the transition pattern in them. These zones are used to facilitate the generalization of the model. Equations (4) to equation (10) reflect the system transitions that occur. In these equations, the number of distribution channels has been generalized to $K$.

Zone I: State $i=R$ :

$P_{R-Q}=\frac{\left[\mu_{1}+\mu_{2}+\cdots+\mu_{K}\right] P_{R}}{\mu_{0}}$

Zone II: State $i=(R-Q)$ :

$P_{i}=\frac{\mu_{0} P_{i-Q}}{\left[\mu_{0}+\mu_{1}+\mu_{2}+\cdots+\mu_{K}\right]}$

Zone IIIIa: From state $i=(R-2 Q)$ to $(R-(m-1) Q)$ :

$P_{i}=\frac{\mu_{0} P_{i-Q}+\left[\mu_{1}+\mu_{2}+\cdots+\mu_{K}\right] P_{i+Q}}{\left[\mu_{0}+\mu_{1}+\mu_{2}+\cdots+\mu_{K}\right]}$

Zone IIIb: From state $i=(N, R-Q)$ to $(N, R-(m-$ 1) $Q)$ :

$P_{i}=P_{R}$

Zone IV: State $i=R-m Q$ :

$P_{i}=\frac{\mu_{0} P_{i-Q}+\left[\mu_{1}+\mu_{2}+\cdots+\mu_{K}\right]\left[P_{i+Q}+P_{N, i+Q}\right]}{\left.\left[\mu_{0}+\mu_{1}+\mu_{2}+\cdots+\mu_{K}\right)\right]}$

Zone V: From State $i=(R-(m+1) Q)$ to $(R-(k-$ 1) $Q)$ : 


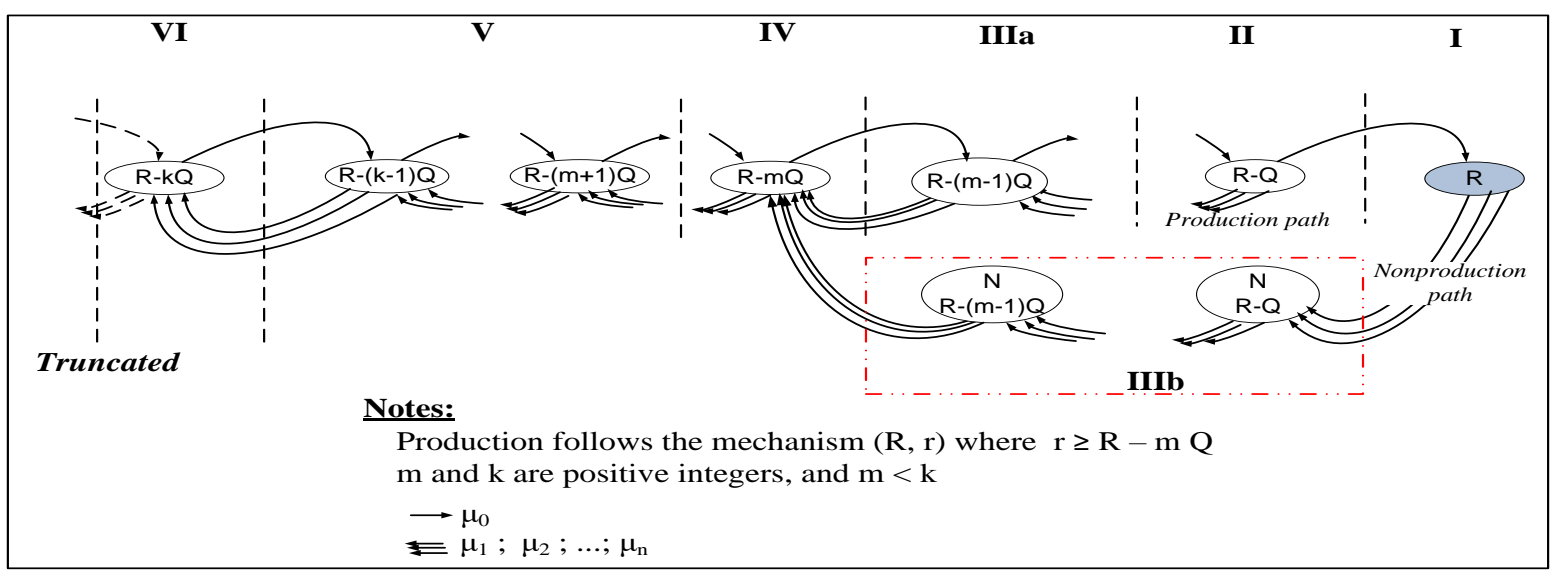

Figure 4. State transition diagram

$P_{i}=\frac{\mu_{0} P_{i-Q}+\left[\mu_{1}+\mu_{2}+\cdots+\mu_{K}\right] P_{i+Q}}{\left.\left[\mu_{0}+\mu_{1}+\mu_{2}+\cdots+\mu_{K}\right)\right]}$

Zone VI: State $i=(R-k Q)$ :

$P_{i}=\frac{\left[\mu_{1}+\mu_{2}+\cdots+\mu_{K}\right] P_{i+Q}}{\mu_{0}}$

We need to calculate the probability of the system being in the state and the system of transition equations (4) to (10), which can be solved numerically. In brief, the algorithm for this case is designed as follows.

\section{STAGE-0: Initialization}

a. For a given number of channels $\mathrm{K}$ and the stock target value $R$ and the delivery lot size $Q$ :

1. Choose a value for $\mathrm{m}$, for example $m=3$

2. Choose a number $k$ (large enough, but not necessarily too large), for example $k=50$

b. Set a deviation tolerance value for each iteration cycle, for example, tol $=0.00001$.

c. Assign an initial value for $P_{i}$ for all $i$, for example $P_{i}=1$ (where $P_{i}$ is the probability of system being in state $i$ ).

d. Save all $P_{i}$ values to $P_{\mathrm{i}, \text { init }}$

STAGE -1: Iteration

Use $P_{R}=1$ before normalization as the anchor of the calculation (it never changes the value). Then, using the most recent probability values as input, compute all $P_{\mathrm{i} \neq \mathrm{R}}$ as follows:

a. Update the value of $P_{i}$ for the state in Zone I using equation (4).

b. Update the value of $P_{i}$ for the state in Zone IIIa using equation (6).

c. Update the value of $P_{i}$ for the state in Zone IIIb using equation (7).

d. Update the value of $P_{i}$ for the state in Zone IV using equation (8).

e. Update the value of $P_{i}$ for the state in Zone $\mathrm{V}$ using equation (9).

f. Update the value of $P_{i}$ for the state in Zone VI using equation (10).

g. Normalize $P_{i}$ so that $\sum P_{i}=1$

h. Save the values of $P_{i}$ as $P_{i, \text { new }}$
STAGE -2: Check wether the convergence of the calculations has been obtained

a. Calculate the calculation deviation, $\operatorname{dev}=$ $\left|P_{i, \text { new }}-P_{i, \text { init }}\right|$

b. Compare whether the calculation deviation from the most recent iteration with the previous iteration, dev, is already less than the tol. If YES, then go to STAGE-3, the iteration is complete. But if NO, then set $P_{i, n e w}=P_{i, \text { init }}$ and repeat the iteration by returning to STAGE-1

\section{STAGE -3: End}

The transition equation for Zone II, namely equation (5), is redundant, hence is not required. Here, $P_{R}$ is chosen as an anchor, i.e., it is set to a spesific value according to stage 0 part (c) and will never be changed or updated before normalization. The response variable average finished good stock (avgFG) then can be calculated.

\section{Results and Discussions}

The system studied consists of factories producing at a specific rate and several distribution channels (more than two). The factory follows the mechanism of $(R, r)$ and delivers its products to the warehouse of finished goods (FG Stock). It is assumed that this storage process occurs on a unit-by-unit basis. The maximum amount of stock in FG Stock is $R$ units. Normally, the minimum stock is $r$ units, but it can be less. The process of delivering products from the factory to each warehouse channel will occur in batches, that is, several $Q$ units (in numerical example discussed: 25 units). Delivery occurs periodically following the inventory cycle time in each warehouse. The cycle time depends on the amount of demand from consumers. All shipments or delivery of $Q$ units are carried out by certain fleets, for example, trucks. Delivery time depends on the distance from the warehouse location to the factory. In this case, the distance can be expressed in units of time. Then, the system is analogous to a single-stage - multi-server queuing system. In a 
Table 2. Comparison of the average value of Finished Good Stock obtained from two models (Numerical vs Simulation)

\begin{tabular}{|c|c|c|c|c|c|c|c|c|c|c|c|c|}
\hline \multirow{3}{*}{$\begin{array}{c}\text { Average } \\
\text { Finished } \\
\text { Good Stock } \\
\text { (avgFG) }\end{array}$} & \multicolumn{12}{|r|}{ average } \\
\hline & Numerical & & & & & & & & & & & 36.36 \\
\hline & Simulation & 47.79 & 39.84 & 35.56 & 36.95 & 41.23 & 34.63 & 41.66 & 38.99 & 31.63 & 36.83 & 38.51 \\
\hline
\end{tabular}

manufacturing context, it is a split system where the split process occurs in the "buffer", not on the "machine" (see [19], for detail).

Modeling with a discrete-event simulation method will be much better, in terms of the depth and

breadth of the entity types discussed, than mathematical or numerical modeling. For example, Salsabila et al. [20] were able to uncover a continuous process problem quite well using discrete simulation modeling. In this case, experiments with multiple replications were required to obtain reasonable inference. Mathematical/numerical performance modeling, on the other hand, will emphasize more a holistic depiction of the case being studied where the results of the analysis can be obtained more straightforwardly. For this reason, the approximate analytical model discussed in this paper is more desirable to obtain. The value of the response variable can be obtained directly without the need for statistical analysis as in the simulation model. However, an analytical model obtained needs to be validated by, for example, comparing its calculation accuracy with the simulation results.

\section{Numerical Example and Comparing with Simulation Result}

Suppose a factory produces with $r=15$ and $R=75$. The rate of production is $\mu_{0}=2.5$ units / time unit. There are three delivery destinations at different locations, namely Warehouse-1, Warehouse-2, and Warehouse-3. Each shipment is transported by truck with a load of 25 units. The distances for each warehouse from the factory are 50, 30, and 15, respectively (which are expressed in units of time). The average inventory cycle times in each warehouse are 50, 30, and 15, respectively (expressed in units of time). The calculation results show that the average amount of inventory in the finished product warehouse is 36.36 units. An equivalent discreteevent simulation model is conducted in the ARENA simulation software. Table 2 summarizes the comparison of the output of the numerical model and the simulation model.

To see whether the difference is significant, a $\boldsymbol{t}$-test is carried out using the following steps:
Step-1: Hypothesis statement:

$H_{o}$ : Numerical result $=$ simulation result (as claim)

$H_{1}$ : Numerical results $\neq$ simulation results

Step-2: At $\alpha=0.05$ with $d . f .=9$, the critical values are: \pm 2.262

Step-3: The t-test value:

$$
t=\frac{\bar{X}-36.36}{s / \sqrt{n}}=\frac{38.51-36.36}{4.49 / \sqrt{10}}=1.514
$$

Step-4: Because the $t$-value is between critical values: $-2.262<1.514<2.26$

then the null hypothesis is accepted.

Step-5: In this case, there is sufficient evidence to support the claim that the numerical model and the simulation model provide the same calculation results for the average FG Stock.

In the numerical model, the delivery process that occurs in batches has been "forced" to occur in units per unit by flattening it in the form of equation (1). However, because the developed transition diagram has accommodated this batch process, the result looks quite satisfactory. Then, the delivery lot size in this discussion must be the same for all channels, i.e., $Q$ units. In a real case, the delivery lot size may differ from one channel to another, so the model cannot be used in general. However, this model can still be used with the equivalent method. This should be the next research topic. The multi-channel situation in the supply chain system is common. Hence, this model will be very useful for completing the problem of modeling complex supply chain systems with the multi-channel distribution.

\section{Conclusions}

A two-echelon supply chain system, namely factories and distributions with multiple channels, can be viewed as a single-stage multi-server queuing system. The branching points to several distribution channels are located in the finished product warehouse. In this case, the factory is assumed to produce $(R, r)$ by the mechanism. The shipment lot size to each channel is the same, which is $Q$ units. The warehouse distance in each channel can differ from one another, where the distance is expressed in units of time. Inventory cycle time in each warehouse may also vary between warehouses. This inventory cycle time will accommodate the arrival 
rate of customer demand, which is not involved in this study.

With markovian analysis, the state transition diagram in the system provides seven zones. The compiled system of equations is solved numerically by setting one of the states (namely state $R$ ) as the anchor of the calculation. It is found that the model which is arranged based on this single-stage queuing model is satisfactory, compared with the discreteevent simulation results.

The model obtained can be used to complement the multi-echelon supply chain system modeling with the multi-channel distribution. However, one assumption of the same lot size for all channels can be relaxed in subsequent studies to be closer to the real system conditions.

\section{Acknowledgment}

The author would like to thank UKWMS for facilitating the research which was the source of this paper. The author also would like to thank the reviewers who perfected this paper.

\section{References}

1. Murdapa, P.S., Pujawan, I N., Karningsih, P.D., and Nasution, A. H., A Numerical Analysis Model Involving Carbon Emissions in a Single Echelon Supply Chain Systems with Two Distribution Channels: A Preliminary Model Proposed, The 6th International Conference of Industrial Engineering and Applications (ICIEA), IEEE, 2019, Waseda, Japan

2. Karaman, A. and Altiok, T.R., Approximate Analysis and Optimization of Batch Ordering Policies in Capacitated Supply Chains, European Journal of Operational Research, 193, 2009, pp. 222-237.

3. Saetta, S., Paolini, L., Tiacci, L. and Altiok, T., A Decomposition Approach for the Performance Analysis of a Serial Multi-echelon Supply Chain, International Journal of Production Research, 50(9), 2012, pp. 2380-2395.

4. Murdapa, P.S., Pujawan, I N., Karningsih, P.D., and Nasution, A. H., Incorporating Carbon Emissions in Queuing Models to Determine Lot Sizes and Inventory Buffers in a Supply Chain. International Journal of Intelligent Enterprise, 7(4), 2020, pp. 373-390

5. Xiong, W. and Altiok, T., An Approximation for Multi-server Queues with Deterministic Reneging Times, Annals of Operations Research, 172, 2009, pp. 143-151.

6. Koroliuk, V. S., Melikov, A. Z., Ponomarenko, L. A., and Rustamov, A. M.,Methods for Analysis of Multi-channel Queueing System with Instantaneous and Delayed Feedbacks, Cybernetics and Systems Analysis, 52(1), 2016, pp.58-70
7. Pryshchepa, O. V. and Lebedev, E. O., On a Multi-channel Retrial Queueing System, Cybernetics and Systems Analysis, 53(3), 2017, pp.441-449

8. Sultan, A. M., Hassan, N. A. and Elhamy, N. M., Computational Analysis of a Multi-server Bulk Arrival with Two Modes Server Breakdown, Mathematical and Computational Applications, 10(2), 2005, pp. 249-259

9. Sultan, A. M., Multi-channel Bi-level Heterogeneous Servers Bulk Arrival Queueing System with Erlangian Service Time, Mathematical and Computational Applications, 12(2), 2007, pp. 97105

10. Ke, J.C., Wu, C.H., and Pearn, W.L., Analysis of an Infinite Multi-server Queue with an Optional Service, Computers \& Industrial Engineering 65, 2013, pp. 216-225

11. Goswami, V., Analysis of Discrete-time Multiserver Queue with Balking, International Journal of Management Science and Engineering Management, 9(1), 2014, pp. 21-32.

12. Liberopoulos, G. and Dallery, Y., Base Stock versus WIP Cap in Single-stage Make-to-stock Production Inventory Systems, IIE Transactions, 34, 2002, pp. 627-636

13. Gershwin, S.B. and Schor, J.E., Efficient Algorithms for Buffer Space Allocation, Annals of Operations Research 93, 2000, pp. 117-144

14. Tan, B., and Gershwin, S. B., Analysis of a General Markovian Two-stage Continuous-flow Production System with a Finite Buffer, International Journal of Production Economics, 120, 2009, pp. 327-339

15. Tan, B., and Gershwin, S. B., Modelling and Analysis of Markovian Continuous Flow Systems with a Finite Buffer, Annals of Operations Research, 182(1), 2011, pp. 5-30

16. Le Bihan, H. and Dallery, Y. A Robust Decomposition Method for the Analysis of Production Lines with Unreliable Machines and Finite Buffers, Annals of Operations Research 93, 2000, pp. 265-297

17. Matta, A., Dallery, Y., and Di Mascolo, M., Analysis of Assembly Systems Controlled with kanbans, European Journal of Operational Research, 166, 2005, pp. 310-336

18. Karaesmen, F. and Dallery, I., A Performance Comparison of Pull Type Control Mechanisms for Multi-stage Manufacturing, International Journal of Production Economics, 68, 2000, pp. 59-71

19. Helber, S., Approximate Analysis of Unreliable Transfer Lines with Splits in the Flow of Material, Annals of Operations Research, 93(14), 2000, pp. 217-243

20. Salsabila, N.Y., Siswanto, N., Widodo, E., and Rochmadhan, O. K., Throughput Analysis on a Multi-stage Manufacturing System, Jurnal Teknik Industri, 21(2), December 2019, pp. 6978. 